Colloids and Surfaces

A: Physicochemical and Engineering Aspects 183-185 (2001) 247-257

\title{
Oxidation of azo dyes in oil-in-water microemulsions catalyzed by metalloporphyrins in presence of lipophilic acids
}

\author{
Maria Häger a, Krister Holmberg b,*, António M. d'A. Rocha Gonsalves c \\ Arménio C. Serra ${ }^{\mathrm{d}}$ \\ a Institute for Surface Chemistry, P.O. Box 5607, SE-114 86 Stockholm, Sweden \\ ${ }^{\mathrm{b}}$ Department of Applied Surface Chemistry, Chalmers University of Technology, SE-412 96 Göteborg, Sweden

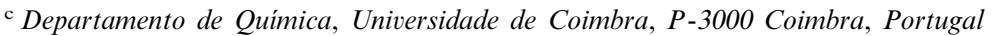 \\ ${ }^{d}$ Universidade Católica Portuguesa, P-3080 Figueira da Foz, Portugal
}

\begin{abstract}
Hydrogen peroxide oxidation of two aqueous azo dyes, methyl orange and amaranth, catalyzed by manganese porphyrins, have been performed in an oil-in-water microemulsion based on a nonionic surfactant, $\mathrm{C}_{12} \mathrm{E}_{8}$. A lipophilic acid was used as cocatalyst in a variable amount. The oxidation was followed by UV spectroscopy. It was shown that the reactions performed in the microemulsion were rapid for both dyes and the reaction rate increased strongly with increasing amount of lipophilic acid added to the reaction mixture. As reference, the same reactions were performed in a two-phase system without surfactant. The reaction profiles in the two-phase system were similar to those in the microemulsion, but the rate was considerably lower. The effect of addition of small amounts of ionic surfactant was also investigated. Addition of anionic surfactant gave a pronounced decrease in reaction rate, whereas a cationic surfactant gave a small reduction in rate. We propose that the rate-limiting step is formation of a metallo-acylperoxy complex at the boundary between oil and water domains. The metallo-acylperoxy complex oxidizes the azo dye in a subsequent step. (C) 2001 Elsevier Science B.V. All rights reserved.
\end{abstract}

Keywords: Surfactant; Microemulsion; Two-phase; Reaction; Oxidation; Hydrogen peroxide; Dye; Porphyrin; Lipophilic acid; Phase behavior

\footnotetext{
* Corresponding author. Tel.: + 46-31-7722969; fax: +4631-160062.

E-mail address: kh@surfchem.chalmers.se (K. Holmberg).
}

\section{Introduction}

Microemulsions based on water, organic solvent and surfactant can be used as reaction media to overcome the incompatiblity problem that can occur in organic synthesis. Macroscopically the 
microemulsions are homogeneous, but in a microscopic view the surfactant film forms structures such as oil-in-water droplets, bicontinuous systems and water-in-oil droplets, depending on the composition and the surfactant used. These small structures with their very large interfacial area enable oil-soluble and water-soluble reactants to meet more frequently compared to a two-phase system based on water and oil which always has a much smaller interfacial area. In some cases, a reaction performed in a microemulsion is even faster than in a two-phase system with added phase transfer catalyst, such as a Q-salt or a crown ether [1,2]. Phase transfer catalysis can also be used in a microemulsion system in which case a further acceleration of the reaction may be obtained [3].

There is a growing concern about degradation of commercial dyes that are toxic to the environment and resistant to biodegradation. Such dyes need to be eliminated from the waste water in textile and other process industries. Most dyes are hydrolytically stable and acid- or basecatalyzed cleavage is usually not a viable approach. The majority of dyes contain oxidizable groups, however, and different methods for oxidation of dyes have been investigated such as treatment with one-electron oxidants [4,5] and photocatalytic processes [6-8].

In this work we have used an oil-in-water microemulsion based on a nonionic surfactant as reaction medium to oxidize two different aqueous azo dyes, methyl orange and amaranth, with hydrogen peroxide as oxygen donor. Different combinations of manganese porphyrins and lipophilic acids were employed as oxidation catalysts. 4-Tert-butylpyridine was used as a combined base and ligand. The effect on the reaction rate of introducing small amounts of an ionic surfactant was investigated. The hydrogen peroxide and the dye were dissolved in the aqueous phase, while the porphyrin, the lipophilic acid and the base were dissolved in the organic phase. As reference, the same reactions were performed in a two-phase system composed of water and a chlorinated solvent.

\section{Experimental}

\subsection{Materials}

The surfactant octa(ethylene glycol)monododecyl ether $\left(\mathrm{C}_{12} \mathrm{E}_{8}\right)$ was purchased from Nikko Chemicals (Tokyo, Japan). The other surfactants used, sodium dodecyl sulfate (SDS) and tetradecyltrimetylammonium bromide $\left(\mathrm{C}_{14} \mathrm{TAB}\right)$, were purchased from BHD Laboratory Supplies (England) and Sigma, respectively. The dyes, methyl orange $(85 \%)$ and amaranth $(80 \%)$, were obtained from Aldrich. The manganese porphyrins used were synthesized elsewhere and given as a gift $[9,10]$. The solvents used, dichloromethane, hexadecane and methanol, were from Merck. Benzoic acid $(99.5 \%)$ and dodecanoic acid $(99.5 \%)$ were from Fluka and Sigma, respectively. The base, 4-tertbutylpyridine (99\%), was obtained from Aldrich. Water was millipore-filtered. All the reagents were employed without further purification. A $5 \%$ hydrogen peroxide solution was prepared from a concentrated one $(30 \%)$ and its $\mathrm{pH}$ adjusted to $4.5-5.0$ with sodium hydrogen carbonate.

\subsection{Microemulsions}

The aqueous component of the microemulsion consisted of dye $(17 \mu \mathrm{mol})$ dissolved in a $5 \mathrm{wt}^{\%} \%$ hydrogen peroxide solution $(2.5 \mathrm{mmol})$. The dye was either methyl orange or amaranth (Fig. 1a) and it was dissolved in the aqueous phase by means of ultra sound. The oil component consisted of a 1:1 mixture of hexadecane and dichloromethane to which a catalytic amount of a manganese porphyrin $(0.5 \mu \mathrm{mol})$ was added together with a lipophilic acid $(10-100 \mu \mathrm{mol})$ and 4-tert-butylpyridine $(50 \mu \mathrm{mol})$. The manganese porphyrins used for the reaction can be seen in Fig. 1(b). Benzoic acid or dodecanoic acid were employed as the lipophilic acid. The microemulsion was formulated with $\mathrm{C}_{12} \mathrm{E}_{8}$. When investigating the effect of ionic surfactant, the anionic surfactant SDS or the cationic surfactant $\mathrm{C}_{14} \mathrm{TAB}$ was added in an amount corresponding to $5 \mathrm{wt}^{\%} \%$ of the total amount of surfactant. 


\subsection{Two-phase systems}

Unless otherwise stated the two-phase systems consisted of dichloromethane-water. The reagents and catalysts used were the same as in the microemulsion systems.

\subsection{Reactions}

The reactions were performed in an oil-in-water microemulsion at room temperature $\left(17-20^{\circ} \mathrm{C}\right)$. The microemulsion consisted of $10 \mathrm{wt} \%$ surfactant, $10 \mathrm{wt} \%$ oil component and $80 \mathrm{wt} \%$ aqueous component. The total amount of the reaction mixture was $2 \mathrm{~g}$. Small amounts of the reaction mixture were taken at different time intervals, diluted in methanol, and analyzed by UV/VIS spectroscopy using a Perkin Elmer Lambda 18 UV/VIS spectrometer.

All the reactions were also performed in a two-phase system based on an aqueous phase and an oil phase with a weight ratio of $8: 1$ under conditions similar to those in the microemulsion. The two-phase system was stirred vigorously. At different time intervals stirring was ceased and

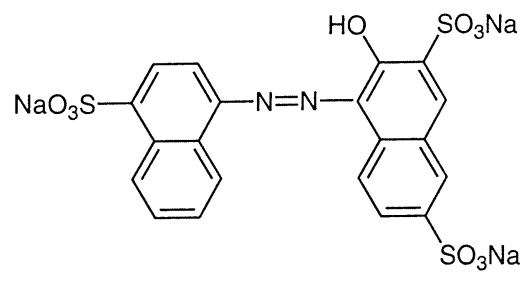

Amaranth samples were taken from the aqueous phase, diluted in methanol and analyzed by UV/VISspectroscopy.

\section{Results}

\subsection{Phase behavior}

Studies of the temperature effect on the phase behavior of the oil-in-water microemulsion (L1 phase) were made in absence and in presence of reactants and catalysts. The pure $\mathrm{C}_{12} \mathrm{E}_{8}-$ dichloromethane-hexadecane-water system gave a microemulsion between 15 and $27^{\circ} \mathrm{C}$. At higher temperatures a lamellar phase was observed. Adding hydrogen peroxide and dye to the system made only marginal changes to the boundaries of the $\mathrm{L} 1$ region. The lower phase boundary was found to be the same and the upper phase boundary decreased to $25^{\circ} \mathrm{C}$. Addition of the other ingredients, i.e. the lipophilic acid, base and manganese porphyrin to the system gave shifted the upper limit of the microemulsion from 27 to $35^{\circ} \mathrm{C}$. This is probably due to the lipophilic acid,

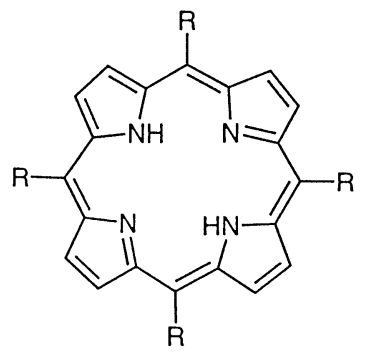

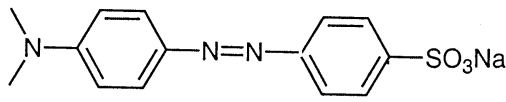

Methyl Orange<smiles>Pc1c(Cl)cccc1Cl</smiles><smiles>CCCNOS(=O)(=O)c1ccc(Cl)c(P)c1Cl</smiles>

2

Fig. 1. The two azo dyes: used as substrates and the two manganese porphyrins; used as oxidation catalysts. 


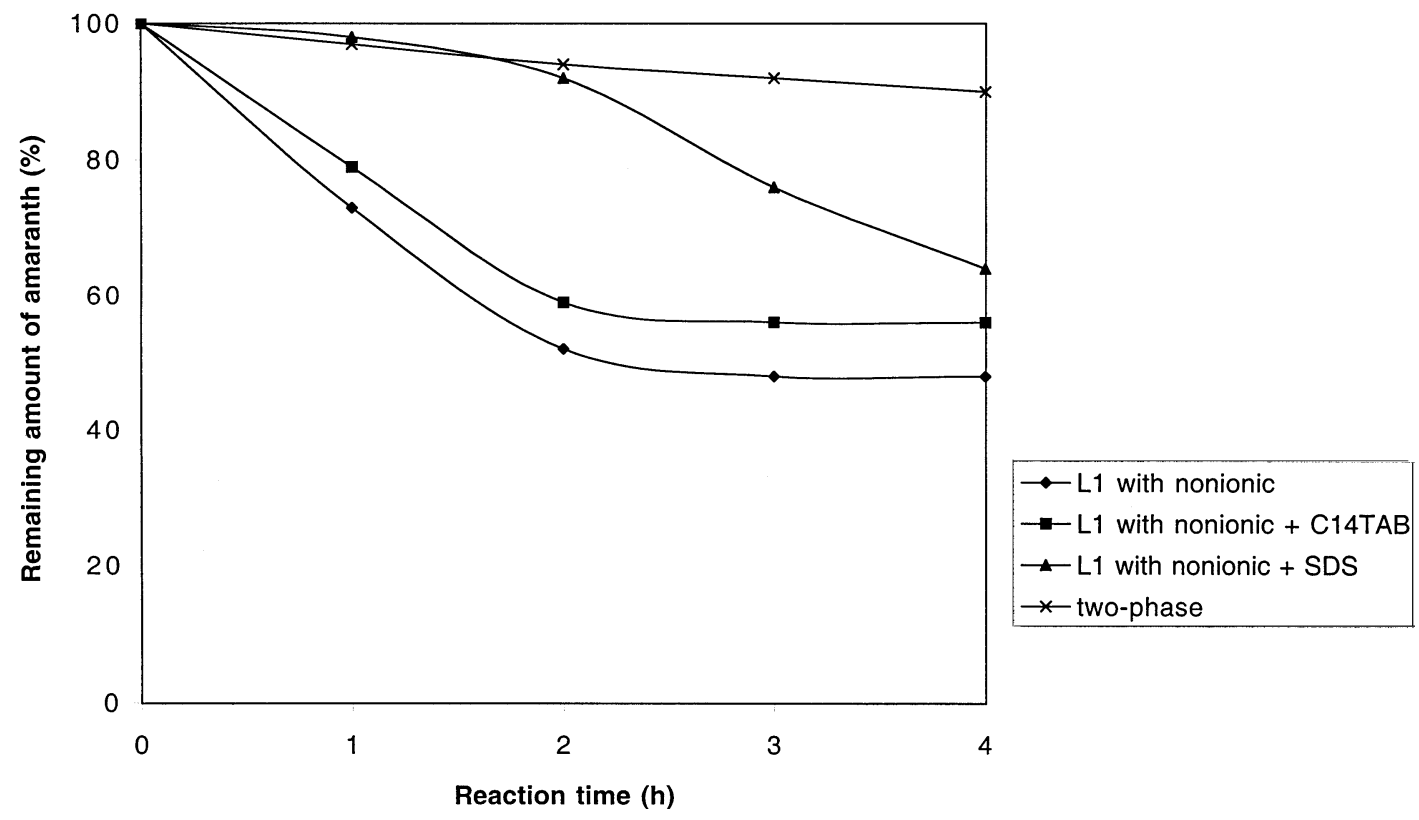

Fig. 2. Reaction profiles for the oxidation of amaranth catalyzed by manganese porphyrin 1 ( 1 equiv.) and cocatalyzed by benzoic acid (20 equiv.) performed in an oil-in-water microemulsion based on $\mathrm{C}_{12} \mathrm{E}_{8}$, hexadecane, dichloromethane and water and in a two-phase (water-dichloromethane) system. The influence of addition of $5 \%$ SDS or $\mathrm{C}_{14} \mathrm{TAB}$, calculated on the total surfactant amount, is shown.

effectively deprotonated by the base, acting as a cosurfactant. The upper temperature limit was difficult to establish and is somewhat uncertain, however, owing to the low boiling point of dichloromethane $\left(40^{\circ} \mathrm{C}\right)$. The phase boundaries of the oil-in-water microemulsion in presence of all reactants and catalysts change during the course of the reaction. The L1 region was shifted towards lower temperatures. This effect was particularly pronounced when the concentration of the lipophilic acid was high. It was also more pronounced in the oxidation of amaranth than of methyl orange.

\subsection{Oxidation of amaranth}

The oxidation of amaranth was performed at room temperature in an oil-in-water microemulsion (L1) based on $\mathrm{C}_{12} \mathrm{E}_{8}$, water, dichloromethane and hexadecane. The reaction was monitored by UV-spectroscopy measuring the decrease of the broad dye peak at $512 \mathrm{~nm}$. Scanning the range from 300 to $800 \mathrm{~nm}$ revealed no product peak appearing after $4 \mathrm{~h}$ reaction. Manganese porphyrin 1 and benzoic acid (20 equivalents with respect to the porphyrin) were used as reaction promoters if not otherwise mentioned. The oxidation reaction performed in the L1 phase gave a $50 \%$ consumption of the dye after $3 \mathrm{~h}$. The effect of small amounts of ionic surfactant added to the microemulsion system was also investigated (Fig. 2). Addition of SDS, which imparts a negative charge to the interface, resulted in a decreased reaction rate. This can be explained by electrostatic repulsive forces preventing either the anion of the lipophilic acid or/and the anionic dye to reach the interfacial region. Also the cationic surfactant $\mathrm{C}_{14} \mathrm{TAB}$ gave a reduction of the reaction rate but the effect was much smaller than for SDS. The retardation caused by the cationic surfactant is an unexpected but similar effects have been reported for other reactions involving $\mathrm{C}_{14} \mathrm{TAB}$ and ionic reagents of opposite charge $[2,11]$. The choice of counterion of the cationic surfactant has been shown to be important for the yield and reaction rate in micellar and microemul- 
sion systems and polarizable ions such as bromide have been found to be particularly effective in preventing approach by anionic reagents to the interface [2,11]. We have no explanation for the fact that none of the reaction went to completion. One may speculate that the products formed inhibit further reaction. As reference, the same reac- tion was carried out in a two-phase system based on water and dichloromethane. As can be seen from Fig. 2, reaction in the two-phase system during $4 \mathrm{~h}$ reaction time was much slower and almost no decay of the dye peak could be observed. The influence of the lipophilic acid on the reaction kinetics was also investigated. As can be
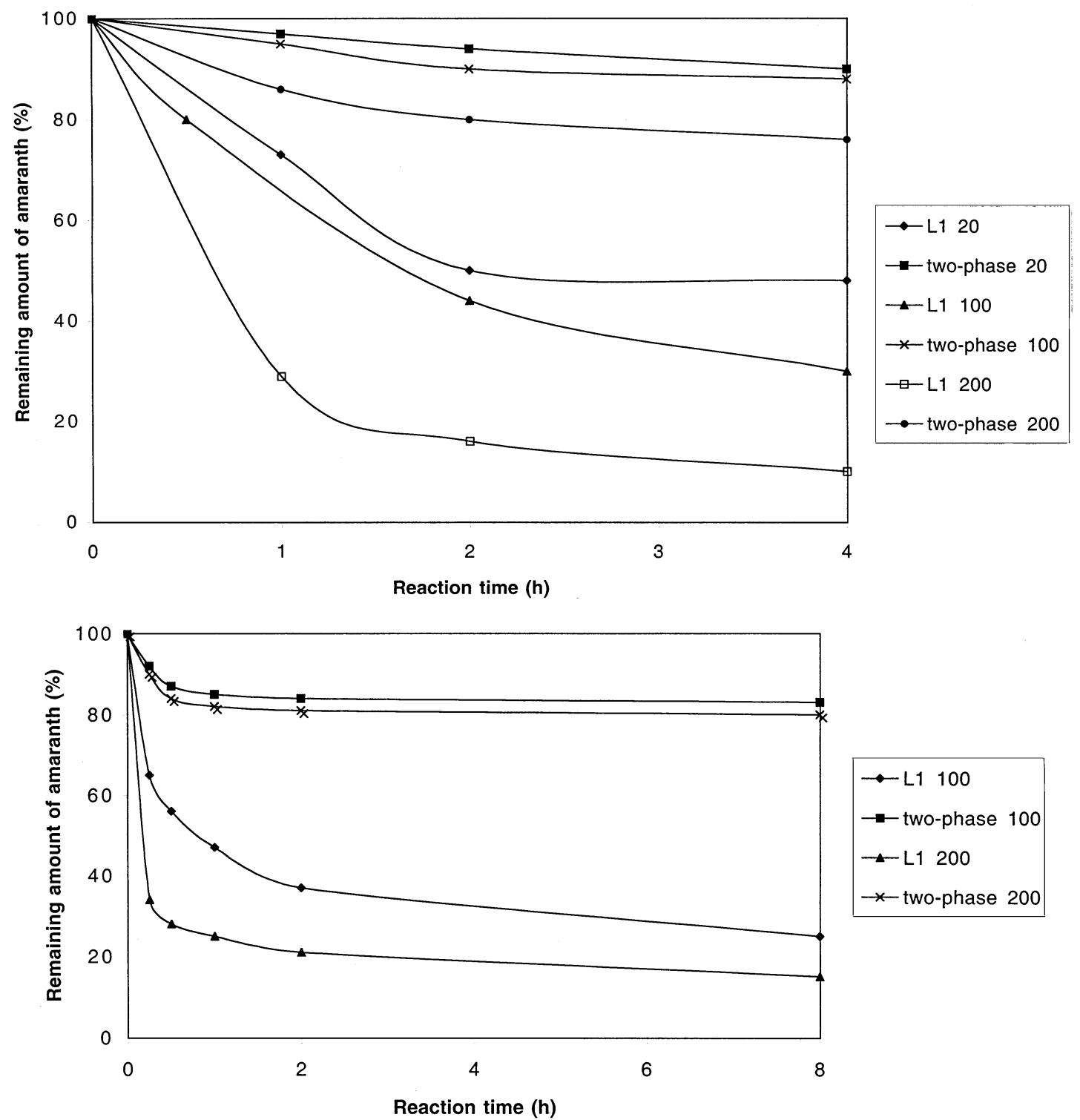

Fig. 3. Reaction profiles for the oxidation of amaranth catalyzed by manganese porphyrin 1 above or 2 below; together with varying amounts of benzoic acid performed in an oil-in-water microemulsion based on $\mathrm{C}_{12} \mathrm{E}_{8}$, hexadecane, dichloromethane and water and in a two-phase (water-dichloromethane) system. The amount of benzoic acid is calculated as equivalents relative to the porphyrin. L1 100 means reaction carried out in the L1 phase using 100 equivalents of benzoic acid relative to the porphyrin, etc. 
seen from Fig. 3(a), the reaction was accelerated with increasing benzoic acid concentration. The reactions performed in the two-phase system were all slow although also here an increasing amount of benzoic acid resulted in increased reaction rate. Replacement of manganese porphyrin $\mathbf{1}$ by manganese porphyrin $\mathbf{2}$ gave a similar trend with overall higher reaction rates (Fig. 3b).

\subsection{Oxidation of methyl orange}

Oxidation of methyl orange was also performed in an oil-in-water microemulsion based on $\mathrm{C}_{12} \mathrm{E}_{8}$, dichloromethane, hexadecane and water. The reactions were monitored by UV spectroscopy by measuring the disappearance of a broad peak at $413 \mathrm{~nm}$, which is characteristic of methyl orange. During the course of the reaction this peak shifted to lower wavelengths. New peaks were being formed at 328 and $251 \mathrm{~nm}$. Reaction profiles for the dye oxidation, catalyzed by manganese porphyrin 1 in presence of benzoic acid (20 equivalents with respect to the porphyrin), and performed in the oil-in-water microemulsion are given in Fig. 4(a). No dye could be detected after $4 \mathrm{~h}$ reaction. Addition of $\mathrm{C}_{14} \mathrm{TAB}$ to the microemulsion based on $\mathrm{C}_{12} \mathrm{E}_{8}$ slowed down the reaction slightly and addition of SDS gave a substantial reduction in rate. The reaction in the two-phase system, water and dichloromethane, was more sluggish and $80 \%$ of the starting material still remained after $8 \mathrm{~h}$. These results are in accordance with the result discussed above for amaranth oxidation. Replacing benzoic acid by dodecanoic acid gave similar reaction profiles, but with all reactions proceeding at a lower rate, which can be seen in Fig. 4(b).

Fig. 5 illustrates the effect of varying amount of benzoic acid on the rate of oxidation of methyl orange. When using manganese porphyrin 2 the reaction rates also increased with increasing concentration of benzoic acid, as is shown in Fig. 5(a). Surprisingly, the reaction performed in the two-phase system based on water and dichloromethane was faster than that in the L1 phase based on nonionic surfactant. This could possibly be due to some reactant being very soluble and reactive in methylene chloride, but this issue was not further investigated. To make a more relevant comparison between the two systems, the oil component of the two-phase system was changed to the 1:1 mixture of dichloromethane and hexadecane that had been used as the oil component of the microemulsion. The reactions in this new two-phase system was considerably slower and again the microemulsion could be seen as a better reaction medium (Fig. 5a). Similar effects were also obtained with manganese porphyrin $\mathbf{1}$ as reaction promoter (Fig. $5 b)$. The microemulsion gave faster reactions than both two-phase systems but the rate increase caused by increasing amount of added acid was more pronounced for the two-phase systems than for the microemulsion.

\section{Discussion}

Metalloporphyrins are known as efficient catalysts for many oxidation reactions [12-15]. A study has previously been made of an alkene epoxidation performed in a two-phase system catalyzed by sulphonated metalloporphyrins. Hydrogen peroxide was used as oxidant and the reaction was carried out in presence of different lipophilic acids. Metalloporphyrin catalyzed oxidations with hydrogen peroxide as oxygen donor need both an axial ligand and a base to attain selectivity of the hydrogen peroxide. The base 4-tert-butylpyridine was used for both purposes. It was proposed that the reaction occured at the aqueous-organic interface where the lipophilic acid and the porphyrin formed some sort of membrane that efficiently activated the oxygen donor [10]. One objective of the present investigation was to increase the understanding of the mechanism of this reaction. Comparison of reaction rates in microemulsions and in two-phase systems which have very different oil-water interfacial areas might provide clues as to the importance of the interface for the reaction kinetics.

Oxidation of azo dyes is normally very $\mathrm{pH}$ sensitive. At a $\mathrm{pH}$ above 5 oxidation of methyl orange occurs at the amino group rather than at the azo group [16,17]), resulting in the formation of amine oxide instead of degradation into small 

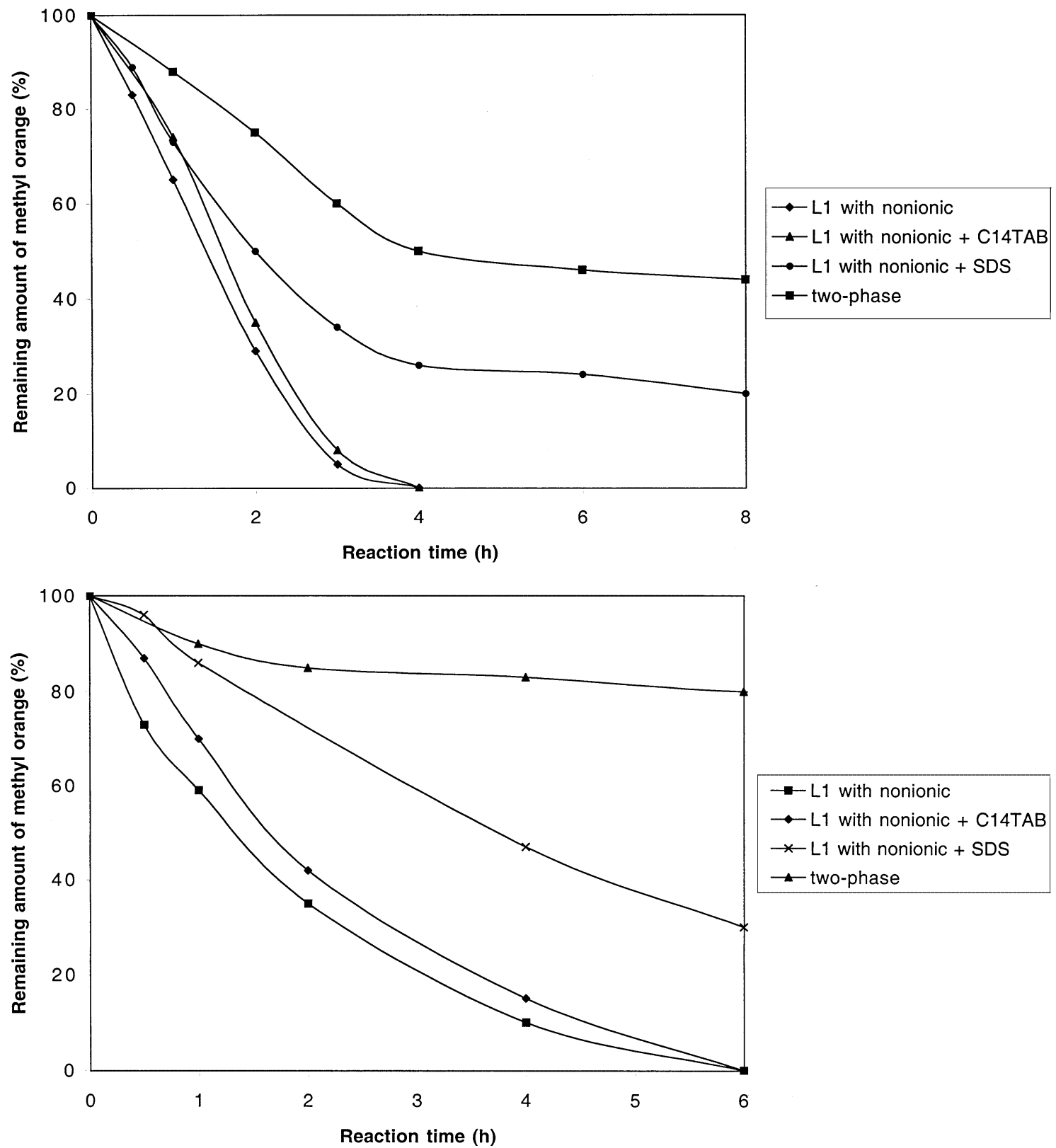

Fig. 4. Reaction profiles for the oxidation of methyl orange catalyzed by manganese porphyrin 1 ( 1 equiv.) and cocatalyzed by: (above) benzoic acid (20 equiv.); or (below) dodecanoic acid (20 equiv.) in the system $\mathrm{C}_{12} \mathrm{E}_{8}$-hexadecane-dichloromethane-water (L1) and in the water-dichloromethane system (two-phase). The effect of addition of $5 \% \mathrm{SDS}$ or $\mathrm{C}_{14} \mathrm{TAB}$, calculated on the total surfactant amount, is shown as well.

fragments of the dye. Still a bleaching takes place as is manifested by a shift of the absorption peak into the UV region [16]. Oxidative attack at the azo group occurs at lower $\mathrm{pH}$, however, when strong oxidizing conditions are used [17,18]. In our case, a hydrogen peroxide solution in combination with the manganese porphyrin at a $\mathrm{pH}$ just below 5 gave an efficient bleaching, as indicated 
by the shift of the absorption peak into lower wavelengths. With amaranth the oxidation resulted in disappearance of absorption in the mea- suring range of $300-800 \mathrm{~nm}$. This can be interpreted as a result of extensive degradation of the dye. No attempts have been made to investi-
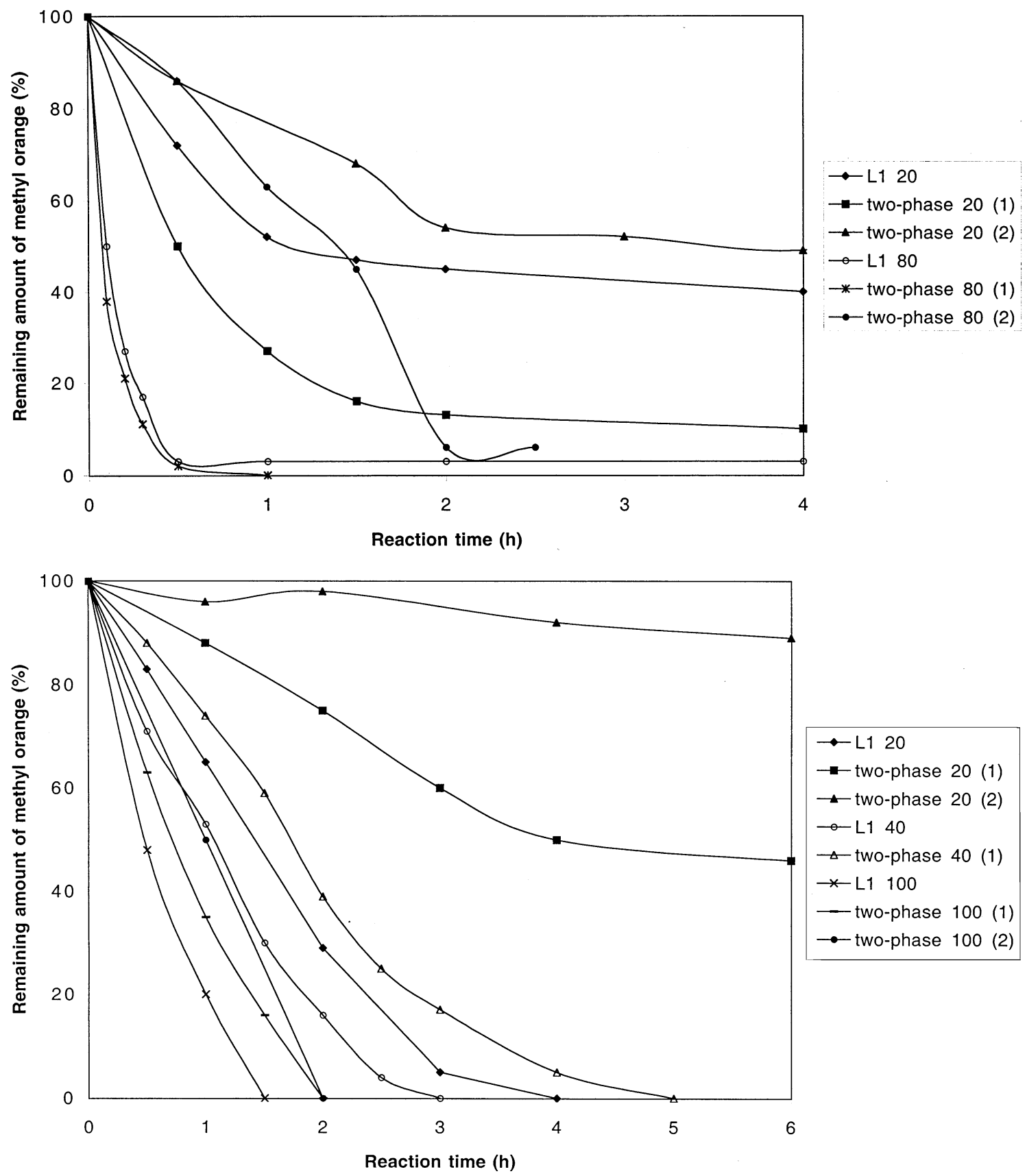

Fig. 5. Reaction profiles for the oxidation of methyl orange catalyzed by manganese porphyrin 2 (above) or $\mathbf{1}$ (below) together with varying amount of benzoic acid. The oxidation reactions are performed in an oil-in-water microemulsion and in a two-phase system based either on dichloromethane-water (1) or dichloromethane-hexadecane-water (2). 


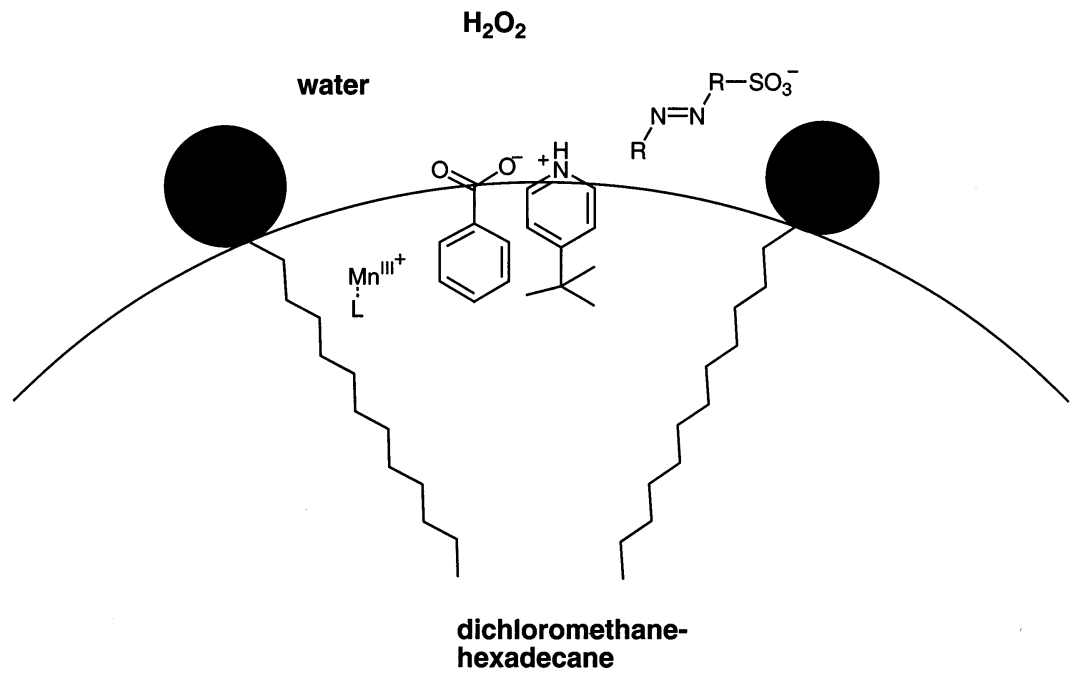

Fig. 6. A schematic illustration of the oil-in-water microemulsion containing the reagents and the catalysts. The system is based on $\mathrm{C}_{12} \mathrm{E}_{8}$, hexadecane, dichloromethane and water. The dye (substrate) and the hydrogen peroxide are dissolved in the aqueous phase and the other ingredients, i.e. the manganese porphyrin, written as $\mathrm{Mn}^{\mathrm{III}}$--- $\mathrm{L}$, the lipophilic acid and the 4-tert-butylpyridine, are dissolved in the oil phase. Benzoic acid is used as lipophilic acid in the figure.

gate the products formed the degradation process. It is known from the literature that oxidative degradation of azo dyes can occur along several pathways [16,19-21].

The reason for selecting azo dyes as substrates for the oxidation reaction is two-fold. First, as mentioned in the introduction, oxidation of azo dyes is an environmentally important issue. Second, previous studies have shown that this oxidation proceeds fairly slowly in a two-phase system; hence an increase in reaction rate would be easy to monitor. The hydrogen peroxide and the dye were dissolved in the aqueous phase and the other compounds, i.e. the manganese porphyrin, the lipophilic acid and the base, were dissolved in the organic phase. Fig. 6 gives a schematic picture of the reagents in the microemulsion. The proposed mechanism is outlined in Fig. 7. Initially, the base deprotonates the lipophilic acid to form the surface-active ammonium salt of the acid. The acid salt, situated at the interface, is then oxidized by hydrogen peroxide to give the corresponding peracid. This oxidation step is likely to be catalyzed by the manganese complex. The oil-soluble peracid formed interacts with the manganese porphyrin to form a metallo-acylperoxy complex which subsequently decomposes to give a metaloxo species and the anion of the peracid. A similar mechanism for the formation of the metal-oxo species has been proposed before [15]. In the proposed reaction scheme the ligand coordinates with the metal atom at the free site followed by a dissociation of the counter anion, which leaves the site free to react with the oxygen donor, i.e. the peracid. A recent study indicates that the metallo-acylperoxo complex is the working oxidant for the dye [22]. This oil soluble species reacts with the somewhat surface active aqueous dye at the oil-water interface.

The reaction profiles for the oxidation of the two dyes, amaranth and methyl orange, were quite similar with the oxidation of methyl orange being faster than that of amaranth. As is implied by the proposed mechanism, an essential part of the oxidation reaction takes place at the interface between the aqueous and the organic phase. The fact that the reactions performed in the microemulsions proceeded at a faster rate than those in two-phase systems point to the importance of a large interfacial area. When the amount of lipophilic acid is increased the reaction is accelerated both in the microemulsion and in the two- 
phase system. This implies that formation of the metallo-acylperoxy complex is a rate limiting step which, in turn, supports the view of the importance of the interface [22] since this complex forms at the oil-water boundary. The importance of the access to the interface for the reagents was also shown by the experiments with added ionic surfactant. As discussed above, both SDS and $\mathrm{C}_{14} \mathrm{TAB}$ made access of the reagents to the interface more difficult resulting in a decreased reaction rate.

\section{Conclusions}

The system used, $\mathrm{C}_{12} \mathrm{E}_{8}$-dichloromethane-hexadecane-water is a good reaction medium for oxidation of azo dyes, providing proper compatibility of the reagents. The reaction medium is, thus, suitable for investigating the role of the aqueous-organic interface. The fact that the reac- tions were considerably faster in the microemulsions than in the two-phase systems strongly suggest that the oil-water interfacial area is crucial for the rate of the oxidation. The fact that increasing amount of lipophilic acid accelerates the reaction while addition of ionic surfactants, which render access of the reagents to the interface more difficult, makes the reaction more sluggish supports the view that the reaction is interface dependant. We propose that the rate limiting step is the formation of the metalloacylperoxy complex at the oil-water interface.

\section{Acknowledgements}

We thank the Foundation for Strategic Research within its program for Colloid and Interface Technology for financing this work. We also thank the Foundation Chalmers University of Technology for a scholarship that gave $\mathrm{MH}$ the<smiles>CC(C)(C)c1ccncc1</smiles>

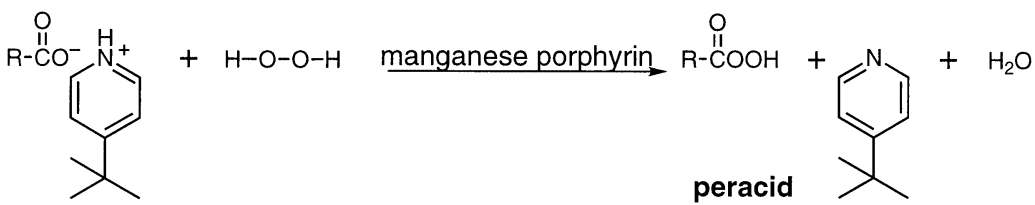
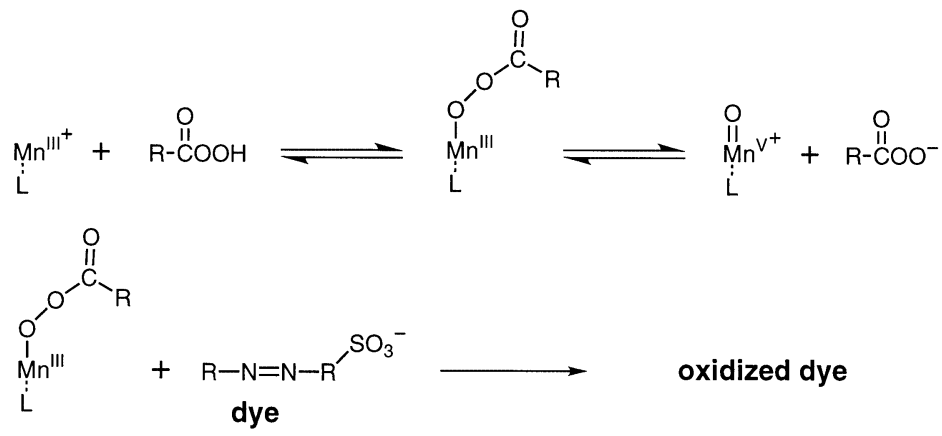

Fig. 7. Proposed mechanism of oxidation of an azo dye (see text). 
opportunity to execute most of the research work presented in this paper at the University in Coimbra.

\section{References}

[1] F.M. Menger, A.R. Elrington, J. Am. Chem. Soc. 113 (1991) 9621.

[2] S. Gutfelt, J. Kizling, K. Holmberg, Colloids Surf. A128 (1997) 265.

[3] M. Häger, K. Holmberg, Tetrahedron Lett. 41 (2000) 1245.

[4] M. Stiborova, B. Asfaw, E. Frei, H.H. Schmeiser, Collect. Czech. Chem. Commun. 61 (1996) 962.

[5] N.H. Ince, D.T. Gonenc, Environ. Sci. Technol. 18 (1997) 179.

[6] K. Vinodgopal, P.V. Kamat, J. Photochem. Photobiol. A83 (1994) 141.

[7] C. Nasar, K. Vinodgopal, S. Hotchandini, A.K. Chattopadhay, P.V. Kamat, Res. Chem. Intermed. 23 (1997) 219.

[8] U. Stafford, K.A. Gray, P.V. Kamat, J. Catal. 167 (1997) 25.
[9] A.M.d'A. Rocha Gonsalves, M.M. Pereira, J.M.T.B. Varejão, J. Heterocycl. Chem. 28 (1991) 635.

[10] A.M. d'A. Rocha Gonsalves, M.M. Pereira, A.C. Serra, An. Quim. Int. Ed. 92 (1996) 375.

[11] S.-G. Oh, J. Kizling, K. Holmberg, Colloids Surf. A104 (1995) 217.

[12] S. Quici, S. Banfi, Pozzi. G. Gazz. Chim. Italy 123 (1993) 579 .

[13] A.M. d'A. Rocha Gonsalves, M.M. Pereira, J. Mol. Catal. 113 (1996) 209.

[14] D. Dolphin, T.G. Traylor, L.L. Xie, Acc. Chem. Res. 30 (1997) 251.

[15] S. Banfi, C. Cavalieri, M. Cacazzini, A. Trebicka, J. Mol. Catal. A: Chem. 151 (2000) 17.

[16] J. Oakes, P. Gratton, J. Chem. Soc. Perkin Trans. 2 (1998) 2563.

[17] L. Pentimalli, Tetrahedron 5 (1959) 27.

[18] N.A. Johnson, E.S. Gould, J. Org. Chem. 39 (1974) 407.

[19] J. Oakes, P. Gratton, R. Clark, I. Wilkes, J. Chem. Soc. Perkin Trans. 2 (1998) 2569.

[20] J. Oakes, P. Gratton, J. Chem. Soc. Perkin Trans. 2 (1998) 2201.

[21] C. Galindo, P. Jacques, A. Kalt, J. Photochem. Photobiol. A130 (2000) 35.

[22] A.M. d' A. Rocha Gonsalves, A.C. Serra, submitted. 